Document downloaded from:

http://hdl.handle.net/10251/99450

This paper must be cited as:

Riera-Guasp, M.; J. Antonino-Daviu; José Roger-Folch; Molina Palomares, MP. (2008). The Use of the Wavelet Approximation Signal as a Tool for the Diagnosis of Rotor Bar Failures. IEEE Transactions on Industry Applications. 44(3):716-726. doi:10.1109/TIA.2008.921432

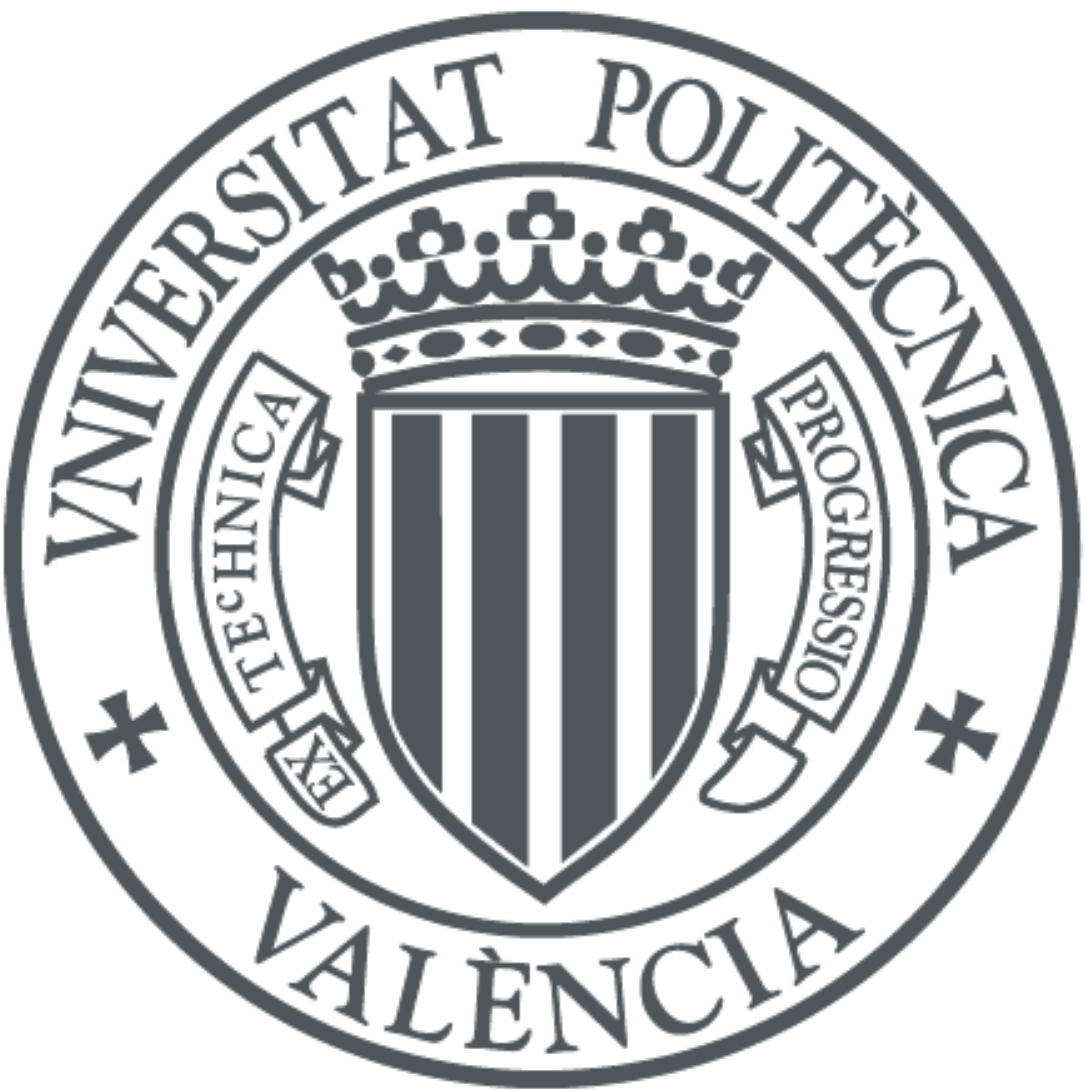

The final publication is available at

http://doi.org/10.1109/TIA.2008.921432

Copyright Institute of Electrical and Electronics Engineers

Additional Information 


\title{
The Use of the Wavelet Approximation Signal as a Tool for the Diagnosis of Rotor Bar Failures
}

\author{
M. Riera-Guasp* \\ mriera@die.upv.es
}

\author{
J.A. Antonino-Daviu* \\ joanda@die.upv.es
}

\author{
J. Roger-Folch* \\ jroger@die.upv.es
}

\author{
M.P. Molina* \\ pimolina@die.upv.es
}

\author{
* Institute of Energetic Engineering; Department of Electrical Engineering; Universidad Politécnica de Valencia \\ P.O.Box 22012; 46071 Valencia, Spain
}

\begin{abstract}
The aim of this paper is to present a new approach for rotor bar failure diagnosis in induction machines. The method focuses on the study of an approximation signal resulting from the wavelet decomposition of the startup stator current.

The presence of the left sideband harmonic is used as evidence of the rotor failure in most diagnosis methods based on the analysis of the stator current. Thus, a detailed description of the evolution of the left sideband harmonic during the startup transient is given in this paper; for this purpose, a method for calculating the evolution of the left sideband during the startup is developed and its results are physically explained.

This paper also shows that the approximation signal of a particular level, obtained from the Discrete Wavelet Transform (DWT) of the startup stator current, practically reproduces the time evolution of the left sideband harmonic during the startup. The diagnosis method proposed here consists of checking if the selected approximation signal fits well the characteristic shape of the left sideband harmonic evolution described in the paper.

The method is validated through laboratory tests. The results prove that it can constitute a useful tool for the diagnosis of rotor bar breakages.
\end{abstract}

Index Terms-Broken rotor bars, fault diagnosis, startup transient, wavelet analysis.

\section{INTRODUCTION}

The diagnosis of rotor asymmetries in asynchronous machines has been deeply studied by many authors; a review of relevant papers in the field of rotor asymmetry diagnosis using steady state approaches can be found in [1]. According to the physical magnitude analysed for monitoring the rotor condition, different diagnosis methods have been proposed; there are approaches based on the frequency domain analysis of stator currents [2-3], fluxes on search coils [4], axial flux on coils concentric with the shaft [5], vibrations [6] or rotor speed [7]. Methods based on the analysis of the spectrum of the stator current (Motor Current Signature Analysis, MCSA) have drawn most of the attention in the industrial environment, since this signal can be obtained directly from the usually already existing current transformers, avoiding the installation of any specific probe or the necessity for any intervention on the machine.

The basis of the MCSA methods can be found in [2]. The rotor asymmetry introduces harmonics with characteristic frequencies in the spectrum of the stator current. The most important is known as the Left sideband harmonic, whose frequency is given by (1).

$$
f_{L}=f(1-2 s)=f\left(\frac{2 \Omega-\Omega_{s}}{\Omega_{s}}\right)
$$

Where $f$ is the supply frequency, $s$ is the slip, $\Omega$ is the rotor speed and $\Omega_{s}$ is the synchronous speed.

The breakage diagnosis approach consists of checking if the ratio between the amplitude of the fundamental harmonic and that of the sideband component is lower than certain values $[8,9]$. The main disadvantages of this method are the load-dependence of the results (the approach is not valid for unloaded or light-loaded machines), as well as the possibility of an erroneous diagnostic when harmonics with frequencies similar to those of the sideband harmonics are present due to other causes (such as voltage or load fluctuations or ballbearing faults) [10].

Moreover, all the approaches based on steady-state analysis have some inherent constraints, such as:

- the impossibility of detecting broken bars in the outer cage of double cage machines [11]

- poor frequency resolution and so, poor reliability in applications in which the load or supply conditions are not strictly uniform [12]

To avoid these disadvantages, some new methods based on the detection of the sideband components during the startup transient have been proposed. It is interesting to remark that, when using approaches based on the transient analysis, the load condition of the induction motor is unimportant [13]; the frequency of the sideband varies within a wide range during the startup process and its amplitude reaches values several times higher than in a stationary regime; and this is true for any load condition. This fact makes the detection of these components easier. On the other hand, these methods need a minimum inertia factor of the system or a minimum startup time [14]. This is necessary to avoid the influence of the electromagnetic transient current which completely masks the sideband component during the earliest stages of the startup. Another limitation of these methods is due to the fact that they are unsuitable for diagnosing machines fed through inverters; in these cases, in comparison with the direct on line startup, the slip remains low during the machine acceleration process and so, the frequency of the sideband remains very close to the main frequency, whereas its amplitude remains 
low. Therefore, approaches based on transient analysis are especially suitable in applications with heavy direct on line startup processes (high inertia factor, long startup time). In fact, these are the cases in which a bar breakage is more likely to occur. Anyway, the limitations and application scope of transient and steady-state approaches are clearly different and, in certain way, complementary.

The main concepts of the diagnosis methods based on the characteristic frequency evolution of the left sideband during the start up were described by Elder et al. [15]: For not too fast startups, once the electromagnetic transient finishes, it can be assumed that the machine accelerates following a succession of stationary regimes with increasing speeds. Under such conditions, the left sideband harmonic continuously evolves, changing its frequency and amplitude. Its frequency evolves in a particular way: it starts being equal to the supply frequency when the machine is switched on. As the rotor accelerates, the frequency drops, reaching $0 \mathrm{~Hz}$ when the slip equals 0.5 . Then, it increases again and nearly reaches the supply frequency, when the steady-state regime is reached.

Toliyat [16] and Penman [17] introduced models of the induction machine which enable transient analysis including the effect of broken bars. Qiu [18] proposed a time-varying frequency spectrum for the study of current during the transient. This spectrum enables observing the spectral components during the startup - in order to detect a possible pattern in their evolution. However, the frequency resolution was not high enough, and this made analysis of the resulting pattern difficult.

The suitability of diagnostic methods based on transient analysis was substantially enhanced with the use of the Wavelet transform, a mathematical tool which is more suitable for analysing transient signals than the Fourier transform. In recent years, some methods based on the wavelet transform have been proposed for the detection of the left sideband component evolution within the startup current.

Burnet, Watson et al. $[19,20]$ evaluated the ability of different signal processing strategies for detecting the nonstationary components due to the rotor asymmetry in the startup current, concluding that the best results are obtained with the Wavelet decomposition. They introduced a simplified approach for detecting the left sideband by analyzing the output of a single pass-band filter, based on the convolution of the current signal with a Gaussian wavelet. In [21], the increase of certain wavelet coefficients resulting from the DWT of the startup current is employed as an indicator of the presence of the left sideband; this method requires a pre-treatment of the current signal to suppress the fundamental component. The method proposed in [22] is based on the use of the Continuous Wavelet Transform combined with the wavelet ridge method, in order to reveal this characteristic frequency component. References [14, 23] describe a characteristic pattern in the low-frequency signals resulting from the DWT of the startup current as evidence of the existence of the left sideband component during this transient; and this pattern is physically explained.

In this paper, a method for the diagnosis of rotor bar breakages based on the application of DWT to the startup current is proposed. The diagnostic is based on comparing the time-evolution of the left sideband component, extracted from the experimental startup current, with its theoretical evolution, which has been previously deduced. It has to be remarked that the method allows the direct observation of the evolution of the left sideband harmonic during startup. This method differs from the approaches commented before, where the left sideband harmonic is detected indirectly by means of the alterations that it produces on different parameters or signals associated with the start up process.

This paper is organized as follows: Section II presents a theoretical analysis of the left sideband harmonic evolution during the startup transient. A detailed description of the harmonic evolution during this transient is given, based not only on the frequency variations (already performed in the literature [15]) but also on the evolution of its amplitude. This section also explains a method for calculating the evolution of the left sideband in a given machine for specific startup conditions. Section III describes a new method, based on the DWT, which enables the extraction of the left sideband harmonic from the primary startup current signal. In Section $\mathrm{IV}$, the proposed method is validated by means of laboratory tests; the approach is applied to different startup currents, obtained from healthy and faulty machines. Finally, Section $\mathrm{V}$ introduces and validates a non-dimensional parameter which enables the quantification of the fault severity and automatic detection. The paper also includes three appendices where some expressions and concepts used in Section II are explained.

\section{EVOLUTION OF THE LEFT SIDEBAND HARMONIC DURING THE STARTUP}

The aim of this section is to explain a method for approximately calculating the evolution of the left sideband harmonic during the startup in a cage motor with a broken bar. To avoid unnecessary complexity, an ideal magnetic circuit (no saturation, no magnetic losses, infinite iron permeability, uniform air-gap) and a uniform current density at the rotor bars are assumed.

\section{A. Determination of the Amplitude of the Left Sideband Harmonic in Stationary Regime}

The effects of broken bars can be analysed using the concept of "fault current" introduced by Deleroi [24]. Analysis of a machine with a broken rotor bar can be made considering the superposition of two configurations: the machine in healthy state, and the machine with a current source in the bar that breaks (fault current). The fault current 
is always equal to the current flowing through the same bar in the healthy machine but in the opposite direction, in such a way that the total current through the bar is null. The fault current flows through the short-circuit rings and the remaining bars, originating a magnetic field in the air-gap (fault field, $B_{F}$ ); this field, superposed on the normal field of the healthy machine, causes alterations in its behaviour; more specifically, it induces the current harmonics in the stator windings that are used for the bar breakage diagnosis.

At steady state, the fault current varies sinusoidally with time at the slip frequency. If $f$ is the supply frequency, and $f_{r}$ is the frequency of the rotor current, then the frequency of the fault current $\left(f_{F}\right)$ is given by:

$$
f_{F}=f_{r}=s \cdot f
$$

The shape of the spatial wave of air gap flux density $B_{F}$ $(\alpha, t)$, produced by the fault field is a stepped bipolar wave, whose amplitude and spectral composition changes cyclically with time.

As stated in $[2,24]$, the spatial wave $B_{F}(\alpha, t)$ can be decomposed as the sum of spatial harmonics with $1,2,3 . . p$ ..n pole pairs; these harmonics have a fixed position with respect to the rotor, but their amplitudes oscillate proportionally with the fault (or rotor) current. By applying Leblanc's theorem to every harmonic field, two series of rotating components with constant amplitude and speed are obtained: a series with the same rotating direction as the rotor $(+)$, and another series with the opposite direction $(-)$. The electrical speed related to the rotor has the same absolute value for all these components. This speed is given by (3):

$$
\omega_{F n \pm}^{r}= \pm 2 \cdot \pi \cdot f_{F}= \pm 2 \cdot \pi \cdot s \cdot f
$$

Where $\omega_{F n \pm}^{r}$ denotes the electrical speed related to the rotor of the rotating component of the fault field with $n$ pole pairs with positive or negative rotating direction.

For analyzing the left sideband harmonic, only the wave with the same number of pole pairs as the machine $(n=p)$, which rotates in the opposite direction to the rotor, has to be considered, since this is the harmonic inducing in the stator windings the voltages with the frequencies given by (1). The amplitude of this rotating wave, for a given slip, is deduced in Appendix I, (AI-16) resulting:

$$
\widehat{B}_{F p-}=\frac{\mu_{0}}{\sqrt{2} \cdot \delta \cdot \pi \cdot p} \boldsymbol{K}_{\boldsymbol{F p}} \cdot I_{r}
$$

where, $\mu_{0}$ is the permeability of free space, $\delta$ the radial airgap length, $\boldsymbol{K}_{\boldsymbol{F}}$ is a complex factor defined by (AI-12), which depends on the slip and the constructive characteristics of the machine; this factor, deduced in appendix I, takes into account the distribution of the bars through the air-gap as well as the way in which the fault current is distributed among the rotor bars (rms value and time phase). $I_{r}$ is the rms value of the current in a phase of the rotor, in the healthy machine. The rotating speed of the spatial wave $B_{F p}$, related to the rotor, is given by:

$$
\Omega_{F p^{-}}^{r}=\omega_{F p^{-}}^{r} / p=-s \cdot 2 \pi \cdot f / p
$$

and its electrical speed, related to the stator $\left(\omega_{F p_{-}}\right)$is given by:

$$
\omega_{F p-}=p \cdot\left(\Omega+\Omega_{F p-}^{r}\right)=2 \pi \cdot f(1-2 s)=2 \pi \cdot f_{L}
$$

The rotating field $B_{F p}$-generates a three-phase emf system $\left(e_{L s}\right)$ in the stator winding, with a frequency $f_{L} \neq f$. Since the stator can be considered short-circuited for any frequency other than the supply frequency [24], a three-phase system of currents with frequency $f_{L}$ (left sideband harmonics) flows through the stator windings, generating a sinusoidal field, with constant amplitude, which rotates synchronously with $B_{F p-}$. Similarly, the field $B_{F p-}$ induces in the rotor bars a polyphase emf system $\left(e_{L r}\right)$ which produces the corresponding poly-phase system of currents and sinusoidal field, rotating also synchronously with $B_{F p-}$.

The phenomena described above show that with regards to the analysis of the left sideband components of the stator current, the induction motor can be considered as a double short-circuited machine (rotor and stator phases in shortcircuit), which rotates at a given and fixed speed (assuming steady-state), and with an imposed rotating field $B_{F p}$ in the air-gap.

Under these conditions, since saturation is not considered, the superposition principle can be applied and the left sideband components can be calculated as the stator phase currents of the double short-circuited machine with a imposed rotating field $B_{F p}$ in the airgap, solving the system of phase equations:

$$
\left[e_{L i}(t)\right]=\left[R_{i}\right] \cdot\left[i_{L i}(t)\right]+\left[L_{\sigma_{i}}\right] \cdot\left[\frac{d i_{L i}(t)}{d t}\right]
$$

Where :

$i$ : is an index that varies from 1 to $m_{r}+3$, being $m_{r}$ the number of rotor bars. This index identifies the phase of the machine $\left(i=1,2,3\right.$ for the stator phases, $i=4,5, \ldots m_{r}+3$ for the rotor phases or bars)

$\left[e_{L i}(t)\right]:$ is a column vector, whose $i$ th element is the emf induced in the $i$ th phase by the field $B_{F p}$ - and the rotating fields generated by the stator and rotor currents that this field induces.

$\left[R_{i}\right]$ : is a diagonal matrix sized $\left(m_{r}+3\right) x\left(m_{r}+3\right)$, whose non null elements are the phase resistances.

$\left\lfloor L_{\sigma i}\right\rfloor:$ is a diagonal matrix sized $\left(m_{r}+3\right) x\left(m_{r}+3\right)$, whose non null elements are the phase leakage inductances.

$\left[i_{L i}(t)\right],\left[\frac{d i_{L i}(t)}{d t}\right]$ are column vectors containing the phase currents induced by $B_{F p}$ - and its derivatives.

The details for solving (7) from an imposed rotating field $B_{F p}$ - in the air-gap are explained in the Appendix II. 


\section{B. Evolution of the Left Sideband Harmonic during a Startup}

Equations (7) have been used in the previous section for analysis at steady state; moreover, these equations also allow the calculation of the currents (and so, the sideband components) during the startup transient, whenever the evolution of the yoke flux vector $\vec{\phi}_{F p-}^{r}(t)$ through the transient has been previously calculated. This space vector, defined by (AI-17), represents the distribution of the yoke flux produced by the fault field component $B_{F p^{-}}$. From (AI12 ) and (AI-17) it can be seen that, for a specific machine, this vector depends on the slip and the rms value of the rotor phase current $I_{r}$. Strictly speaking, $I_{r}$ can only be defined for a stationary regime. However, during a not too fast startup, once the electromagnetic transient after the connection extinguishes, the machine evolves approximately following a succession of stationary regimes. For each time, the rotor currents approximately constitute a poly-phase system, so that an rms value can be assigned, using (8):

$$
I_{r}(t)=\sqrt{\frac{1}{m_{r}} \cdot \sum_{i=1}^{m_{r}} i_{r i}^{2}(t)}
$$

Where $i_{r i}(t)$ designates the instantaneous value of the fundamental component of the $i$ th phase current of the rotor. To obtain a realistic evolution of the yoke flux vector $\vec{\phi}_{F p-}$ during the startup transient, a numerical model of the induction machine was used for calculating the evolution of the speed and rotor currents. For this purpose, any model able to calculate these magnitudes during the startup would be suitable; for simplicity, the startup was simulated using the standard induction machine SI units block of the MATLAB/SIMULINK library. Table I gives the parameters used for the simulation. These parameters correspond to the $1.1 \mathrm{~kW}$ industrial cage motor used for the experimental validation (see section IV) and were obtained from conventional tests and from the manufacturer's information.

Fig. 1 shows the process followed for the calculation of the yoke flux vector $\vec{\phi}_{F p-}$ during startup.

TABLE I

MACHINE DetaIls AND PARAMETERS USEd IN THE SimULATION

\begin{tabular}{|c|c|}
\hline Rotor type: & Squirrel-cage \\
\hline Referente frame: & stationary \\
\hline $\begin{array}{r}\text { Nom.power (VA) } \\
1870\end{array}$ & $\begin{array}{c}\text { L-L volt (Vrms) } \\
400\end{array}$ \\
\hline $\begin{array}{cc}\text { Stator } & \text { Rs (ohm) } \\
10.30\end{array}$ & $\begin{array}{c}\text { LIs (H) } \\
0.02\end{array}$ \\
\hline $\begin{array}{c}\operatorname{Rr}(\mathbf{o h m}) \\
5.8 \\
\end{array}$ & $\begin{array}{l}\mathbf{L I} \mathbf{r}(\mathbf{H}) \\
0.02\end{array}$ \\
\hline \multicolumn{2}{|c|}{$\begin{array}{l}\text { Mutual inductance } \mathbf{L m}(\mathbf{H}) \\
0.385 \\
\end{array}$} \\
\hline $\begin{array}{c}\text { Inertia } \mathbf{J}\left(\mathbf{k g m}^{2}\right) \\
0.25\end{array}$ & $\begin{array}{cc}\text { friction factor } F(N m s) & \text { pair of poles } p \\
0 & 2 \\
\end{array}$ \\
\hline Initial conditions: & $\begin{array}{l}\text { s() th(deg) isa,isb,isc (A) pha,phb,phc(deg) } \\
\begin{array}{cccccccc}1 & 0 & 0 & 0 & 0 & 0 & 0 & 0\end{array}\end{array}$ \\
\hline Simulation parame & $\begin{array}{clc}\text { heters: } \text { stop time(s) } & \text { fixed step size(s) } & \text { solver opt. } \\
2 & 0.0002 & \text { ode4(Runge-Kutta) } \\
\end{array}$ \\
\hline
\end{tabular}
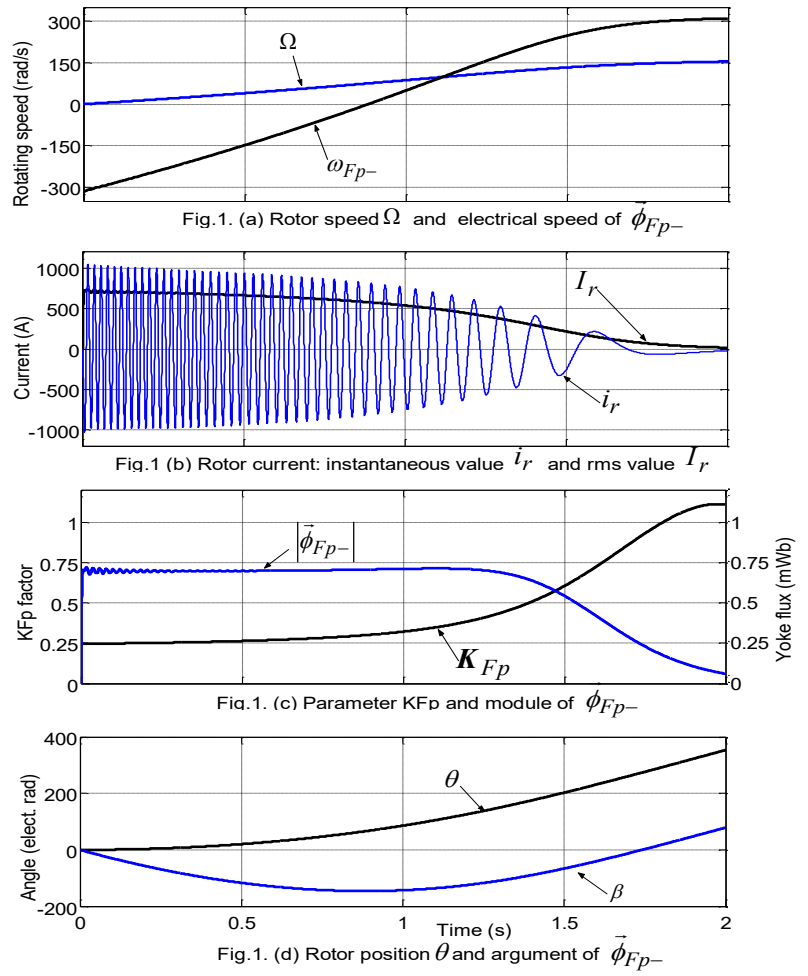

Fig.1 Process for the calculation of the vector $\vec{\phi}_{F p-}$ during startup

Fig. 1(a) and Fig. 1(b) show the evolution of the rotor speed $\Omega(t)$ and current in one of the rotor phases $i_{r i}(t)$; they were obtained by numerical simulation as explained previously. Fig. 1(b) also represents the evolution of the rms value of the rotor currents $I_{r}(t)$. This was obtained by applying (8) to the calculated rotor currents; Fig. 1(c) shows the factor $\boldsymbol{K}_{F p}$, deduced in Appendix I, which introduces the influence of the slip on the distribution of the fault current through the rotor bars and so, in the amplitude of the fault field component $B_{F p^{-}}$. This graph was obtained from the calculated rotor speed $\Omega(t)$ and (AI-12); the evolution of $\boldsymbol{K}_{F p}$ with the slip is physically explained in Appendix III. Fig. 1(c) also shows the evolution of the module of the yoke flux vector $\vec{\phi}_{F p^{-}}$. It was obtained by substituting the calculated values of $I_{r}$, and $\boldsymbol{K}_{F p}$ in (AI-17). Fig. 1(a) shows the evolution of the electrical speed of this vector, $\omega_{F p-}(t)$, in the stator reference frame. It was obtained from $\Omega(t),(1)$ and (6). Finally, Fig.1(d) shows the evolution of $\beta(t)$ (argument of the yoke flux vector $\vec{\phi}_{F p-}$ ) and $\theta(t)$ (position of the rotor in the reference frame of the stator); these magnitudes were calculated by integration of the electrical speed of the yoke flux vector and that of the rotor:

$$
\begin{gathered}
\beta(t)=\int_{0}^{t} \omega_{F p-} d t \\
\theta(t)=\int_{0}^{t} p \cdot \Omega d t
\end{gathered}
$$


Once the yoke flux vector $\vec{\phi}_{F p-}$ is known for each time during the startup, the system (7) can be numerically integrated. Fig. 2 shows the evolution of the left sideband harmonics during the startup transient, in one of the phases of the stator; and computed following the procedure described above.

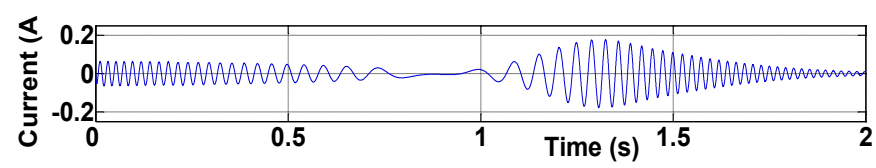

flg. 2.Evolution of left sideband harmonics during the startup (simulation)

Basically, the left sideband harmonics evolve as sinusoidal waves whose frequency and amplitude vary continuously, following characteristic patterns. The frequency decreases continuously from when the machine is connected $(t=0)$, becoming null when the rotor speed equals $\Omega_{\mathrm{s}} / 2(t \approx 0.85 \mathrm{sec}$. see Fig.1(a) and Fig.2). From this point, the frequency of the sideband components increase again, keeping a constant value when the stationary regime is reached; this evolution is according to (1), although this expression was deduced for steady state conditions.

The amplitude of the left sideband also shows a characteristic evolution. Initially, from $t=0$ the amplitude decreases, becoming zero when the frequency becomes null. During the second half of the startup $(t>0.85 \mathrm{sec}$.) the left sideband amplitude initially grows, reaching a maximum which exceeds the amplitudes reached during the first half of the startup. This behaviour can be explained taking into account the evolution of the factor $\boldsymbol{K}_{\boldsymbol{F}}$, which always increases when the slip reduces (see Fig.1c and Appendix III) and also by the Lenz law: the fault field component $B_{F p}$ produces variations of flux linkages at the short-circuited windings of rotor and stator; so, these windings react by generating currents that tend to compensate the magnetic field which causes the flux variations. During the first part of the startup, the contribution of the rotor windings to the compensation of the fault field component is important because, as it is shown by (5), when the slip is high, the bars are exposed to rapid variations of flux. On the other hand, during the second part of the startup, the slip is lower and so the flux linkage variations in the rotor are lower. The contribution of the rotor to the compensation of the fault field component is then irrelevant. The stator currents have to accomplish the compensation by themselves, and so, they need to be higher.

Finally, the amplitude of left sideband components reduces when the startup process finishes. This decrease is caused by the reduction of the amplitude of the fault field component - due to the reduction of the amplitudes of the fundamental components of the rotor currents.

The features described for the amplitude of the left sideband are consistent with the results published by several authors. Williamson and Smith [25], provide a graph for the "theoretical variation of amplitude of the $/ 2 s-1 / f$ component of stator current with rotor speed". Although this graph is deduced at steady state condition, its general shape fits well the description given in this section. The existence of a maximum in the sideband amplitude, during the second half of the startup was also establish by Burnett et al. [19]; in that work the continuous wavelet transform was applied to experimental startup currents of faulty machines; from the observation of the resultant three-dimension graphs, the authors state that "as the non-stationary components travel back towards the $50 \mathrm{~Hz}$ component, in the later stages of the transient, the amplitude of the sidebands pass through a peak value". References [14,23] introduce a method for diagnosing bar breakages based on the analysis of the high level wavelet signals resulting from the DWT of the startup current. When analysing a faulty machine, the evolution (trip) of the sideband component through the frequency bands of the detail signals (first decreasing and later increasing) produces two groups of oscillations in every detail signal, arranged in a characteristic mode. It can also be observed that the oscillations corresponding to the interval where the frequency increases (placed at the second half of the startup) tend to reach larger amplitudes than those corresponding to the decreasing zone.

\section{EXTRACTION OF THE LEFT SIDEBAND HARMONIC USING THE DWT}

This section explains the procedure for extracting the left sideband harmonic from the stator startup transient current, by means of the DWT.

Conceptually, the level $n$ DWT decomposition, of a sampled signal $i_{s}(t)=\left(i_{1}, i_{2}, i_{3} \ldots . . i_{q}\right)$, consists of a digital filtering process with $n+1$ stages. The signal is passed through $n$ pass band filters and a low pass filter. Resulting from this multiple filtering process, $n$ vectors of detail coefficients $\beta^{\mathrm{j}}=\beta^{\mathrm{j}}$ (j: decomposition level $1 \leq j \leq n ; \quad 1 \leq i \leq q \cdot 2^{-j}$ ), and a vector of approximation coefficients $\boldsymbol{\alpha}^{\mathrm{n}}=\alpha^{\mathrm{n}_{i}}$ are obtained. Actually, the process for obtaining the DWT, is made using a recursive algorithm of high computational efficiency denominated "Mallat's algorithm" or pyramidal algorithm [26,27].

From these coefficients, the signal can be reconstructed using the inverse transform (IDWT). The reconstructed signal consists of the sum of $\mathrm{n}$ detail signals and an approximation signal, each one containing the same number of samples, $\mathrm{q}$, as the original signal:

$$
i_{S}(t)=d_{1}+d_{2}+\ldots+d_{n}+a_{n}
$$

An analysis of the procedure for obtaining the reconstructed signal shows that every signal resulting from the decomposition contains the components of the original signal included in a particular frequency band. The detail $d_{j}$ 
contains the information concerning the signal components whose frequencies are included in the interval $\left[2^{-\left({ }^{+}+1\right)} \cdot f_{s}, 2^{-\mathrm{j}} \cdot f_{s}\right]$ Hz. The approximation signal $a_{n}$ includes the low frequency components of the signal, belonging to the interval [0, $\left.2^{-(\mathrm{n}+1)} \cdot f_{s}\right] \mathrm{Hz}[28]$.

If the supply frequency $f$ is included in the detail $d_{n f}$, then the approximation of this level $\left(a_{n f}\right)$, only contains signal components with frequencies below $f$. In the case of healthy machines, these components are negligible if compared with the low frequency oscillations produced by the electromagnetic connection transient. Once this transient is finished, $a_{n f}$ remains almost null.

If a bar breakage exists, the left sideband harmonic has a significant amplitude throughout the startup process; since its frequency is always below $f$, it causes a significant increase in the energy of the approximation signal $a_{n f}$ during the startup process; in Section IV it will be shown that the evolution of $a_{n f}$ over time follows a pattern similar to that described in Section II for the left sideband harmonic. This enables a very reliable diagnosis of bar breakages.

The value of the decomposition level $n_{f}$ depends on the sampling frequency, and this can be easily calculated from condition (12) that specifies that the upper limit of the frequency interval of $a_{n f}$ is lower than the supply frequency:

$$
2^{-\left(n_{f}+1\right)} \cdot f_{s}<f
$$

Therefore, the decomposition level of the approximation signal which includes the left sideband harmonic, is given by:

$$
n_{f}=\text { Integer }\left[\frac{\log \left(f_{s} / f\right)}{\log (2)}\right]
$$

\section{VALIDATION OF THE METHOD}

The method was validated by means of laboratory tests performed on two different machines: a standard $1.1 \mathrm{~kW}$ squirrel cage motor and a laboratory machine. Each machine was tested under both healthy and faulty conditions.

The experimental test-bed used (Fig.3) was very simple: the startup current was captured with a digital oscilloscope, using a current transformer (relation 15/5, precision class 0.5 ) and a shunt $(6 \mathrm{~A} / 60 \mathrm{mV}$, precision class 0.5$)$. The current signal was then transferred to a PC and finally analyzed by applying the DWT. For this purpose, the MATLAB wavelet toolbox was

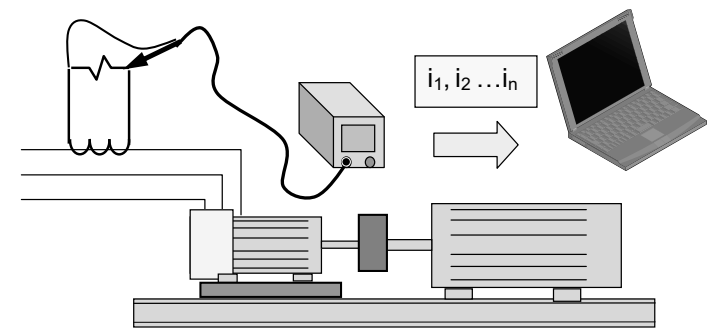

Fig. 3. Experimental test-bed used. For the application of the DWT, Daubechies-44 was selected as the mother wavelet. The reasons for selecting such a high order mother wavelet was to avoid overlapping between adjacent wavelet signals, as explained [14, 28].

\section{A. Commercial Squirrel Cage Motor Tests}

The characteristics of the tested motor are: rated power $1.1 \mathrm{~kW}$, rated voltage: $400 \mathrm{~V}$ (star), 4 poles, rated speed: 1410 rpm and 28 rotor bars. The motor was directly coupled to a D.C. machine. The induction motor was started direct on line, the excitation and armature windings of the D.C. machine were kept open (no load startup). The total inertia of the group amounts to $\mathrm{J}=0.25 \mathrm{~kg} \cdot \mathrm{m}^{2}$.

For capturing the startup current, a sampling rate $f_{s}=5000$ samples/s was used; according to (12), the approximation signal with level $n_{f}=6\left(a_{6}\right)$ is that containing the left sideband harmonic. More accurately, this approximation contains the part of the sideband component with frequency below the upper limit of the frequency band corresponding to $a_{6}$, given by:

$$
f_{u p}=f_{s} \cdot 2^{-\left(n_{s}+1\right)}=5000 \cdot 2^{-(6+1)}=39.0625 \mathrm{~Hz}
$$

Fig. 4 shows the startup primary current and the level-6 approximation signal, corresponding to a test under healthy conditions. After the test, the rotor of the machine was removed and a bar breakage was forced; this was achieved by drilling a hole in the connection point between the bar and the short-circuit ring. Finally, the machine was re-assembled, and tested again. Fig. 5 shows the startup primary current measured under these conditions, and the corresponding level-6 approximation signal. It can be clearly observed that, in the case of the machine with a broken bar (Fig.5), large oscillations appear within the level-6 approximation signal. They do not occur within the corresponding signal for the healthy machine.

Moreover, in the case of the faulty machine, it can be observed that the evolution of the frequency and amplitude of

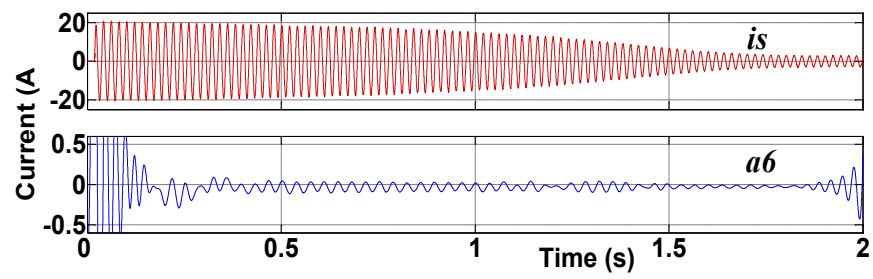

Fig.4.Tested startup current $i_{s}$ and level 6 approximation $a 6$ for the case of healthy machine (test of a commercial machine)

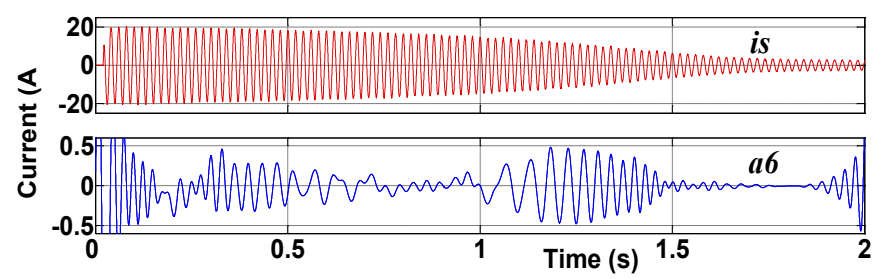

Fig.5.Tested startup current $i_{s}$ and level 6 approximation signal $a 6$ for the case of one broken bar (test of a commercial machine) 
the oscillations of the approximation signal $a_{6}$ matches the evolution of these magnitudes for the left sideband harmonic, calculated in Section II (Fig. 2): It can be seen that frequency decreases progressively, becoming null, and then increases again. Its amplitude also decreases initially, becoming null at $t \approx 0.9 \mathrm{sec}$. and then increases, reaching a maximum at $t \approx$ $0.125 \mathrm{sec}$.

It has to be remarked that the similarity between the $a_{6}$ approximation signal (Fig.5) and the theoretical sideband waveform (Fig. 2) is not achieved at the beginning $(t<$ $0.21 \mathrm{sec}$.) and during the last stages $(t>1.63 \mathrm{sec}$.) of the startup. As commented before, this is because during these periods, the frequency of the sideband component is higher than the upper limit of the frequency band $f_{u p}$ associated with the approximation signal. So, these parts of the sideband component are not included within the approximation signal under study.

B. Laboratory Machine Tests: These tests were performed using a laboratory machine, with very different constructive characteristics from those of the motor tested previously. This is a universal laboratory machine with $p=1$ and twelve 66turns coils (coil pitch 11/12) at the secondary winding (placed at the stator); the endings of each coil are accessible; and so, they can connected to simulate a cage configuration. The primary winding (placed at the rotor) is a balanced threephase, delta-connected winding. This configuration enables easy testing of the resultant induction machine under different fault conditions, achieved by opening $1,2,3 \ldots$ stator coils corresponding to $2,4,6 \ldots$ broken bars. For these tests a sampling frequency $f_{s}=2000 \mathrm{samples} / \mathrm{s}$ was used; according to (13) and (14), for this frequency, the level of the approximation signal which contains the left sideband harmonic is $n_{f}=5$, and the upper limit of its frequency band is $f_{u p}=31.25 \mathrm{~Hz}$. Fig. 6 shows the result of the test for the healthy machine, and Fig. 7 for the machine with two broken bars (a stator coil opened). In both figures, the upper graph

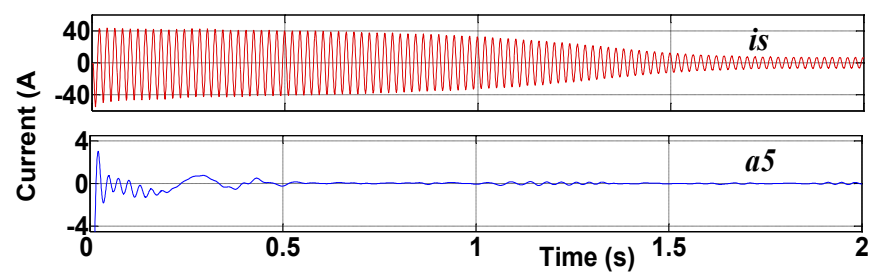

Fig.6.Tested startup current and level 5 approximation signal a5 for the case of a healthy machine (laboratory machine test)

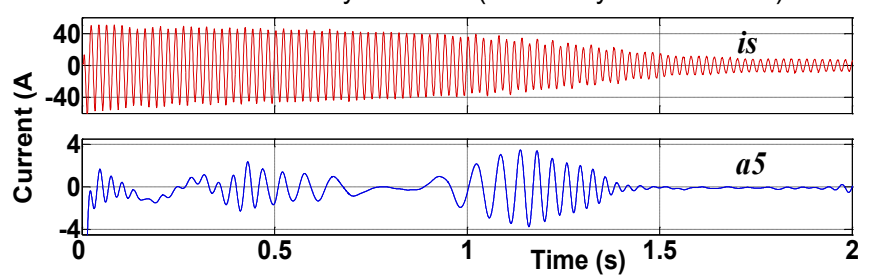

Fig.7.Tested startup current and level 5 approximation signal $a 5$ for the case of a faulty machine (laboratory machine test) corresponds to the startup line current, whereas the waveform below is the level 5 approximation signal resulting from the DWT of the startup line current.

When comparing between both approximation signals, the same phenomena described for the case of the cage motor can be observed: a clear perturbation appears within the approximation signal of the faulty machine. This perturbation does not exist when the machine is tested under healthy conditions. Moreover, the frequency and amplitude of this perturbation fit well the patterns described in the Section II for the theoretical evolution of the left sideband component.

It is noticeable the similitude of the results of the tests for both machines, in spite of their different constructive characteristics and parameters used for calculating the DWT. This fact confirms the validity of the physical bases from which the approach is derived.

In any case, the tests show that the analysis of the approximation is a tool sufficiently sensitive to clearly diagnose a rotor fault, even when only one bar is broken. The fault diagnoses achieved by this approach are highly reliable, because it is very unlikely that a fault, or operating condition other than a rotor asymmetry, could produce a perturbation within the approximation signal with amplitude and frequency following a pattern similar to that described in Section II.

\section{V.QUANTIFICATION OF THE DEGREE OF SEVERITY OF THE FAULT}

Additional tests were performed on both machines with an increasing number of consecutive broken bars; the figures are not included here due to space considerations. The results of these tests show that, when the number of successive broken bars increases, the shape of the approximation signal $a_{n f}$ remains practically unchanged (that is, fits that of the left sideband) - but the amplitude of the oscillations increase. This suggests that the energy of this approximation signal could be a suitable magnitude for defining a parameter for quantifying the severity of the fault. This parameter can be defined as:

$$
\gamma_{n f}(d B)=10 \cdot \log \left[\frac{\sum_{j=N b}^{N s} i_{j}^{2}}{\sum_{j=N b}^{N s}\left[a_{n f}(j)\right]^{2}}\right]
$$

Where $i_{j}$ is the value of the $j$ th sample of the current signal; $a_{n f}(j)$ is the $j$ th element of the order $n_{f}$ approximation signal; $N_{s}$ is the number of samples of the signal, until a steady-state regime is reached; and $N_{b}$ is the number of samples between the origin of the signals and the extinction of the large oscillations within the approximation signal, due to the electromagnetic transient produced immediately after the connection and the border effect. This non-dimensional parameter $\gamma_{n f}$ represents the ratio between the energy of the current signal and that of the approximation signal $a_{n f}$ within the referred time interval, expressed in $\mathrm{dB}$. Table II shows the 
variation of this parameter for both tested machines and for differing numbers of broken bars.

When the number of consecutive broken bars increases, a significant reduction in this parameter can be observed. So this parameter would be suitable for the quantification of the severity of the faults and thus, for possible implementation of automatic bar breakage detection systems.

TABLE II

VARIATION OF THE PARAMETER $\gamma_{n F}$ WITH THE NUMBER OF BROKEN BARS

\begin{tabular}{|c|l|c|c|}
\hline Machine & Status & $\gamma_{n f}(\boldsymbol{d B})$ & $\Delta \gamma_{n f}(\boldsymbol{d B})$ \\
\hline Cage motor & Healthy & $\mathbf{4 4 . 9}$ & - \\
\hline Cage motor & 1 broken bar & $\mathbf{3 5 . 1}$ & $\mathbf{- 9 . 8}$ \\
\hline Cage motor & 2 broken bars & $\mathbf{2 9 . 3}$ & $\mathbf{- 1 5 . 6}$ \\
\hline Lab.machine & Healthy & $\mathbf{3 9 . 5}$ & - \\
\hline Lab.machine & 2 broken bars & $\mathbf{2 5 . 8}$ & $\mathbf{- 1 3 . 7}$ \\
\hline Lab.machine & 4 broken bars & $\mathbf{2 1 . 1}$ & $\mathbf{- 1 8 . 4}$ \\
\hline
\end{tabular}

\section{CONCLUSIONS}

The paper proposes a physically based approach for the diagnosis of rotor asymmetries in induction machines. The method is based on the application of the Discrete Wavelet Transform to the startup stator current. It is shown that a given approximation signal resulting from the DWT of the stator startup current (whose level depends on the sampling rate), practically reproduces the evolution of the left sideband harmonic during the startup process. The similitude between the shape of this approximation signal and the characteristic waveform of the left sideband harmonic during the startup (also deduced in this paper) is reliable evidence of a rotor fault; an expression providing the level of this approximation signal as a function of the sampling rate is deduced.

To facilitate the evaluation of the shape of the approximation signal, this paper includes an analysis of the evolution of the left sideband harmonic during startup; from this analysis, an approximated method for calculating the evolution of this harmonic in a given machine is proposed and also, a general description of its waveform and main characteristics is given and explained.

As a complement of the qualitative diagnosis method proposed, a non-dimensional parameter is introduced to facilitate preliminary fault analysis and the automatic fault detection.

The proposed method is validated by testing a commercial induction motor and a laboratory machine. The results of the tests show that this approach is sensible enough for a clear and reliable diagnosis of the rotor fault, even when only one bar is broken.

\section{APPENDIX I: Deduction of the expressions of $K_{F p} B_{F p-}$, $\vec{\Phi}_{F p}$ as functions of the constructive parameters and the \\ slip:}

For a given slip, the amplitudes of the fault field components depend on the amplitude of the fault current, (or equivalently, on the rms value of the rotor currents). In addition, they also depend on the way in which the fault current is distributed among the rotor bars.

The distribution of the fault current through the rotor bars as a function of the slip and of the machine parameters was deduced by Deleroi [24]; Fig.AI-1 shows the equivalent circuit for the fault current distribution in a rotor cage with $N$ bars, in which "b0" is the broken bar.

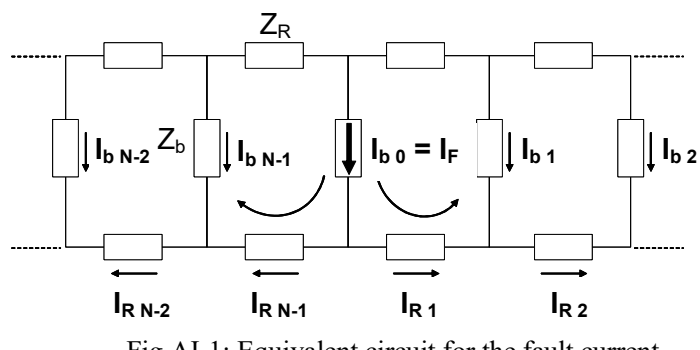

Fig.AI-1: Equivalent circuit for the fault current

In this circuit:

$\boldsymbol{Z}_{\boldsymbol{R}}=R_{R}+j s \cdot X_{\sigma R}$; impedance of a portion of ring between two consecutive bars.

$\boldsymbol{Z}_{\boldsymbol{b}}=R_{b}+j s \cdot X_{\sigma b}$; impedance of a bar.

Ibo: time phasor of the current injected in the broken bar, equal to the fault current (this is, equal to the bar current in the healthy machine, but with the opposite sign).

Ibi: time phasor of the current in the $i$ th bar

$\mathbf{I}_{\mathbf{R i}}$ : time phasor of the current in the $i$ th portion of the ring.

Deleroi proved that:

$$
\frac{\mathbf{I}_{\mathbf{R i}+\mathbf{1}}}{\mathbf{I}_{\mathbf{R i}}}=\mathbf{d}=\frac{\mathbf{I}_{\mathbf{b i}+\mathbf{1}}}{\mathbf{I}_{\mathbf{b i}}}
$$

where $\mathbf{d}$ is a complex number (damping factor) which depends on the machine characteristics and on the slip, defined by (AI-2 ), (AI-3 ), (AI-4)

$$
\begin{array}{r}
\mathbf{d}=(1+\mathbf{A})-\sqrt{(1+\mathbf{A})^{2}-1} \\
\mathbf{A}=\frac{R_{R}+j s\left(X_{\sigma R}+\frac{1}{2} \cdot X_{m_{r}}\right)}{R_{b}+j s \cdot X_{\sigma b}} \\
X_{m r}=\mu_{0} \cdot \frac{b_{t} \cdot \ell}{\delta} \cdot 2 \cdot \pi \cdot f
\end{array}
$$

$X_{m r}$ is the mesh reactance of the equivalent circuit and $b_{t}$ the tooth pitch.

Choosing a suitable time origin:

$$
\mathbf{I}_{\mathbf{b} 0}=-I_{r} \cdot e^{j 0}
$$

Being $I_{r}$ the rms value of the current in a rotor bar, for the slip $s$.

Assuming symmetry in the cage with respect to the broken bar:

$$
\begin{aligned}
& \mathbf{I}_{\mathbf{R} \mathbf{1}}=\mathbf{I}_{\mathbf{R N}-\mathbf{1}}=-\frac{1}{2} \mathbf{I}_{\mathbf{r}} \\
& \mathbf{I}_{\mathbf{R i}}=\mathbf{I}_{\mathbf{R N}-\mathbf{i}}
\end{aligned}
$$


The current through each bar (see Fig.AI-1) can be calculated as:

$$
\mathbf{I}_{\mathbf{b i}}=\mathbf{I}_{\mathbf{R i}+\mathbf{1}}-\mathbf{I}_{\mathbf{R i}}
$$

From (AI-1), (AI-6), (AI-7), results:

$$
\mathbf{I}_{\mathbf{b i}}=\mathbf{I}_{\mathbf{b N}-\mathbf{i}}=(\mathbf{d}-\mathbf{1}) \cdot \mathbf{d}^{i-1} \cdot \frac{\left(-I_{r}\right)}{2}
$$

Once the currents through the bars have been calculated, the next step consists of calculating the space vector of the $n$th order component of the current density wave generated by the fault field; using the formulation of the space vector theory [29], the $n$th order current space vector generated by $\mathrm{b}_{0}$, is given by:

$$
\vec{i}_{F n, 0}=\left[i_{b 0}(t)\right] \cdot e^{j n \cdot 0}=\left[-\sqrt{2} \cdot I_{r} \cdot \cos (s \cdot \omega t)\right] \cdot e^{j 0}
$$

The expression within brackets is the magnitude of the vector, which oscillates in time; its argument is constant and coincides with the coordinate of $b_{0}$ which has been selected as the origin of angular coordinates.

The current space vector produced by every two bars symmetrically placed with respect b0 is given by:

$$
\begin{aligned}
& \vec{i}_{F n, i}+\vec{i}_{F n, N-i}=\left[i_{b i}(t)\right]\left(e^{j n \cdot i \cdot \alpha_{s}}+e^{-j n \cdot i \cdot \alpha_{s}}\right) \cdot e^{j 0}= \\
& =\left[(1-\mathbf{d}) \cdot \mathbf{d}^{i-1} \cdot \sqrt{2} \cdot \frac{I_{r}}{2} \cdot \cos (s \cdot \omega t) \cdot 2 \cdot \cos \left(n \cdot i \cdot \alpha_{s}\right)\right] \cdot e^{j 0}
\end{aligned}
$$

where $\alpha_{\mathrm{s}}$ is the rotor slot pitch, in radians. The resultant space vector of the $n$th wave of current density is obtained by adding the space vector produced by each bar:

$$
\begin{aligned}
\vec{i}_{F n}=\sum_{i=0}^{N-1} \vec{i}_{F n, i}= \\
=\left[\sqrt{2} I_{r} \cos (s \omega t)\left(-1+(1-\mathbf{d}) \sum_{i=1}^{(N-1) / 2} \mathbf{d}^{i-1} \cos \left(n \cdot i \cdot \alpha_{s}\right)\right)\right] e^{j 0}= \\
=\left[\sqrt{2} \cdot I_{r} \cdot \cos (s \cdot \omega t) \cdot \boldsymbol{K}_{\boldsymbol{F n}}\right] \cdot e^{j 0}=\boldsymbol{K}_{\boldsymbol{F n}} \cdot \vec{i}_{F n, 0}
\end{aligned}
$$

where:

$$
\boldsymbol{K}_{\boldsymbol{F n}}=\left(-1+(1-\mathbf{d}) \cdot \sum_{i=1}^{(N-1) / 2} \mathbf{d}^{i-1} \cdot \cos \left(n \cdot i \cdot \alpha_{S}\right)\right)
$$

the complex factor $\boldsymbol{K}_{\boldsymbol{F} n}$ takes into account the distribution of the cage bars along the air-gap and also the way in which the fault current is distributed through the rotor bars (rms value and time phase). The interpretation of this factor is similar to that of a conventional winding factor, since it enables the calculation of the total order $n$ component of the current density wave (or of the yoke flux wave) from that created by a single conductor; but in this case, its value not only depends on the constructive parameters of the machine, but also on the slip. It could be called "order $n$ winding factor of the fault field", for a given slip.
Once the space vector $\vec{i}_{F n}$ is calculated, the space vector theory [29] determines the amplitude of the order $n$ component of the spatial wave of flux density of the fault field:

$$
\begin{aligned}
\hat{B}_{F n} & =\frac{\mu_{0}}{\pi \cdot \delta \cdot n}\left|\vec{i}_{n}\right|=\frac{\sqrt{2} \cdot \mu_{0} \cdot I_{r} \cdot \boldsymbol{K}_{\boldsymbol{F n}}}{\pi \cdot \delta \cdot n} \cdot \cos (s \cdot \omega t)= \\
& =\frac{\mu_{0} \cdot I_{r} \cdot \boldsymbol{K}_{\boldsymbol{F n}}}{\sqrt{2} \cdot \pi \cdot \delta \cdot n} \cdot\left(e^{j s \omega t}+e^{-j s \omega t}\right)
\end{aligned}
$$

The order $n$ spatial vector of yoke flux of the fault field, is obtained [29], as the product of the $n$th order unit inductance per conductor $\left(L_{u n}\right)$ and the space vector of current density $\vec{i}_{F n}$ :

$$
\begin{aligned}
\vec{\phi}_{F n} & =L_{u n} \cdot \vec{i}_{n}=L_{u n} \cdot\left[\sqrt{2} \cdot I_{r} \cdot \cos (s \cdot \omega t) \cdot \boldsymbol{K}_{\boldsymbol{F n}}\right] \cdot e^{j 0} \\
& =\left[L_{u n} \cdot \boldsymbol{K}_{\boldsymbol{F n}} \cdot \frac{I_{r}}{\sqrt{2}}\right] \cdot\left(e^{j s \omega t}+e^{-j s \omega t}\right)
\end{aligned}
$$

where:

$$
L_{u n}=\frac{D \ell \mu_{0}}{2 \pi \delta n^{2}}
$$

being $D$ the average diameter of the airgap and $\ell$ the axial length of magnetic circuit.

Equations, (AI-13), (AI-14) show that the fault field, as stated in $[2,24]$, is built adding the components with $1,2 \ldots p . . n$ pole pairs; each component has a constant position related to the rotor, but an oscillating amplitude. The arguments of the current density and yoke flux spatial vectors of all the components coincide, and they are equal to the angular coordinate of the broken bar; this means that the current density and yoke flux spatial waves of all the components have a maximum at this point. The time phases of these vectors depend on their order (because $\boldsymbol{K}_{\boldsymbol{F} n}$ does); and this means that the components with different orders reach their maximums at different times and so, the fault field varies not only its amplitude, but also its spectra (or shape) cyclically.

Equations (AI-13) and (AI-14) also show that every component of the fault field can be decomposed as the sum of two rotating fields with constant amplitudes and opposite directions (Leblanc's theorem). Considering the negative rotating direction components and particularizing $n=p$ in (AI-13) and (AI-14), result:

$$
\begin{gathered}
\hat{B}_{F p-}=\frac{\mu_{0} \cdot I_{r} \cdot \boldsymbol{K}_{\boldsymbol{F p}}}{\sqrt{2} \cdot \pi \cdot \delta \cdot p} \\
\vec{\phi}_{F p-}^{r}=\left[L_{u p} \cdot \boldsymbol{K}_{\boldsymbol{F p}} \cdot \frac{I_{r}}{\sqrt{2}}\right] \cdot e^{-j s \omega t}
\end{gathered}
$$

These expressions give the amplitude of the flux density wave and the yoke flux vector of the fault field component that induces the left sideband harmonics; the superscript $r$ indicates that the yoke flux vector is related to the rotor reference frame. 


\section{APPENDIX II: Calculation of the left sideband component in steady-state}

Using the formulation of the space vector theory [29], the emfs in (7) can be calculated as:

$$
e_{L i}=N_{c i} \cdot \operatorname{Re}\left(\frac{d \vec{\phi}_{p}}{d t} \cdot \vec{k}_{i}^{*}\right)
$$

where:

$N_{c i}$ is the number of conductors in series of $i$ th phase

$\vec{k}_{i}^{*}$ is the conjugate of the complex winding factor of $i$ th phase

$\vec{\phi}_{p}$ is the resultant, order $p$, space vector of the yoke flux related to the same reference frame than $\vec{k}_{i}$. It will be denoted as $\vec{\phi}_{p}^{s}$ when related to the stator, and as $\vec{\phi}_{p}^{r}$ when related to the rotor reference frame.

For calculating a stator phase emf $(i \leq 3)$, the resultant yoke flux vector is calculated as the sum of the corresponding space vectors generated by the fault field, the stator currents and the rotor currents referred to the stator reference frame:

$$
\vec{\phi}_{p}^{s}=\vec{\phi}_{F p-}^{s}+\vec{\phi}_{L s}+\vec{\phi}_{L r} \cdot e^{j \theta}
$$

where:

$\theta$ is the coordinate (in electrical radians) of the reference frame of the rotor related to the stator reference frame:

$$
\theta=p \cdot \Omega \cdot t
$$

$\vec{\phi}_{L r}$ is the yoke flux vector produced by the rotor currents

$$
\vec{\phi}_{L r}=L_{u p} \cdot \sum_{k=4}^{m r+3} N_{c k} \cdot \vec{k}_{k} \cdot i_{k}
$$

$\vec{\phi}_{L s}$ is the yoke flux vector created by the stator currents (this is, by the left sideband components)

$$
\vec{\phi}_{L s}=L_{u p} \cdot \sum_{k=1}^{3} N_{c k} \cdot \vec{k}_{k} \cdot i_{k}
$$

$\vec{\phi}_{F p-}^{s}$ is the yoke flux vector originated by the fault field component $B_{F p-}$, related to the stator reference frame, which is calculated from (AI-17):

$$
\vec{\phi}_{F p-}^{s}=\vec{\phi}_{F p-}^{r} \cdot e^{j \theta}=L_{u p} \cdot K_{F p} \cdot \frac{I_{r}}{\sqrt{2}} \cdot e^{\beta}
$$

$\beta$ (electrical radians) is the argument of this vector, which varies with time:

$$
\beta=(-s \omega+p \Omega) \cdot t=\omega_{F p-} \cdot t
$$

For calculating the rotor emfs. ( $i \geq 4)$ the yoke flux has to be related to the rotor reference frame:

$$
\vec{\phi}_{p}^{r}=\vec{\phi}_{F p-}^{r}+\vec{\phi}_{L s} \cdot e^{-j \theta}+\vec{\phi}_{L r}
$$

For a specific machine and stationary regime (characterized by the slip or rotor speed), the rms value of the rotor current $I_{r}$ can be calculated; then, using (AI-12), (AI-17), (AII-3) and (AII-6) the vectors $\vec{\phi}_{F p-}^{r}, \vec{\phi}_{F p-}^{s}$ are determined. Finally, the left sideband components can be obtained by numerically solving the equation system (7)

\section{APPENDIX III: Variation of the factor $K_{F p}$ with the slip}

The factor $\boldsymbol{K}_{\boldsymbol{F}}$ depends on the slip through the damping factor d. Fig.1(c) shows the variation of $\boldsymbol{K}_{\boldsymbol{F}}$ when the slip varies from 1 to 0 . This curve was computed from (AI-2), (AI-3 ), (AI-4 ), (AI-12) using the parameters of the $1.1 \mathrm{~kW}$ motor tested in Section IV.

The factor increases as the slip decreases. This behaviour can be explained by means of (AI-2), (AI-3) and (AI-4): When the slip is low, $\mathrm{A}$ is small $(\mathbf{A}<<1)$ and the damping factor $\mathbf{d} \approx 1$. This means that the fault current injected at the broken bar distributes quite equitably among the other bars. As an example, Fig. AIII-1(a) is a qualitative representation of the current density space vector diagram for this case, in a bipolar machine with $N=12$ bars, at a certain time; the current density vectors generated by the bars $1,2 \ldots \mathrm{N}-1$ constitute approximately a balanced star, giving a sum almost null. So, the resultant current density vector approximately equals that produced by the bar $\mathrm{b}_{0}: \vec{i}_{F p} \approx \vec{i}_{F p, 0} \rightarrow \boldsymbol{K}_{\boldsymbol{F p}} \approx 1$.

On the other hand, if the slip is high, then $\mathbf{A} \geq 1$, and the damping factor $\mathbf{d}$ tends to zero and so, as indicated (AI-1), the fraction of the fault current circulating through a certain bar is substantially lower than that flowing through the previous bar. This means (see (AI-8)) that most of the fault current injected from the broken bar circulates throughout the adjacent bars $\left(b_{1}, b_{N-1}\right)$, leading to the vector diagram of Fig.AII-1(b), in which the current density vector generated by $b_{0}$ is practically compensated by those produced by $b_{1}$ and $\mathrm{b}_{\mathrm{N}-1}$.
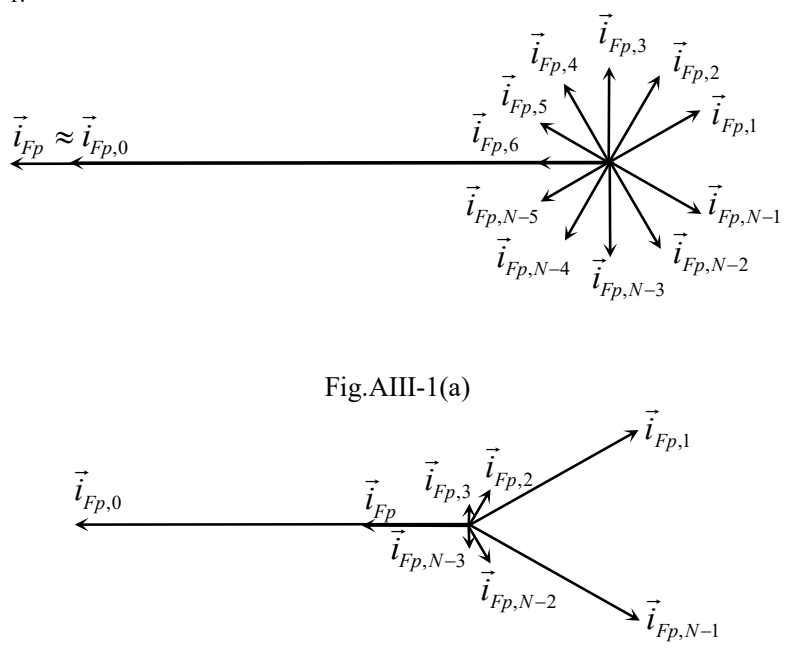

Fig.AIII-1(b)

Fig.AIII-1: Current density vector diagram for the cases: a) low slip $\left(\boldsymbol{K}_{F p} \approx 1\right)$; b) high slip $\left(\boldsymbol{K}_{F p}<<1\right)$

In this way, the resultant current density vector is substantially lower than in the previous case:

$\vec{i}_{F p}<<\vec{i}_{F p, 0} \rightarrow \boldsymbol{K}_{\boldsymbol{F p}}<<1$ 


\section{REFERENCES}

[1] S. Nandi, H.A. Toliyat, "Condition monitoring and fault diagnosis of electrical motors- a review", IEEE Trans. Energy Conversion, vol. 20, no. 4, pp.719-729, December 2005

[2] G.B. Kliman, R.A. Koegl, J. Stein, R.D. Endicott, M.W. Madden, "Noninvasive detection of broken rotor bars in operating induction motors", IEEE Trans. Energy Conversion, vol. 3, no. 4, pp 873-879, December 1988

[3] N.M. Elkasabgy, A.R. Eastham, G.E. Dawson,"Detection of broken rotor bars in the cage rotor on an induction machine", IEEE Trans. Industry Applications, vol. 28, no. 1, pp 165-171, January/February 1992

[4] M.F. Cabanas, F.Pedrayes, M.R. González, M.G. Melero, C.H. Rojas, G.A. Orcajo, J.M. Cano, F. Nuño "A new electronic instrument for the early detection of broken rotor bars in asynchronous motors working under arbitrary load conditions," Proceedings of the 5th IEEE International Symposium on Diagnostics, Electric Machines, Power Electronics and Drives SDEMPED 2005, Vienna, Austria, pp. 29-34, September 7-9, 2005.

[5] J. Penman,A.J. Tait, W.E. Bryan, "Condition monitoring of electrical drives," IEE Proceedings B, vol. 133, no. 3, pp. 142-148, May 1986.

[6] P. Jover, A. Belahcen, A. Arkkio, "Signatures of electrical faults in force distribution and vibration pattern of induction motors", IEE Proc. Electrical Power Applications Volume 153, Issue 4, pp. 523-529, July 2006.

[7] F. Filippetti, G. Franceschini, C. Tassoni, P. Vas, "AI techniques in induction machines diagnosis including the speed ripple effect", IEEE Trans. on Industry Applications, vol. 34, no. 1, pp 98-108, January/february 1998.

[8] A. Bellini, F. Filippetti, G. Franceschini, C. Tassoni, and G.B. Kliman, "Quantitative evaluation of induction motor broken bars by means of electrical signature analysis," IEEE Trans. on Industry Applications, vol. 37, no. 5, pp. 1248-1255, September/October 2001.

[9] M.F. Cabanas, M.G. Melero, and G.A. Capolino, "A new methodology for applying the FFT to induction machine on-line diagnosis," in Proceedings of the International Symposium on Diagnostics for Electrical Machines, Power Electronics and Drives (SDEMPED'99), Gijon (Spain), pp. 537-543., September 1999

[10] R.R. Schoen and T.G. Habetler. "Evaluation and implementation of a system to eliminate arbitrary load effects in current-based monitoring of induction machines," IEEE Trans. on Industry Applications, vol. 33, no. 6, pp. 1571-1577, November/December 1997.

[11] J.F. Watson, S. Elder, "Transient analysis of the line current as a fault detection technique for 3-phase induction motors", Proceedings of the International Conference on Electrical Machines, ICEM'92, Manchester, pp. 1241-1245, September 1992

[12] M. Riera-Guasp, J. A. Antonino-Daviu, J. Rusek, J. Roger-Folch, "Low frequency filtering for the diagnosis of cage asymmetries in induction machines", "Proceedings of the International Conference on Electrical Machines, ICEM 2006 (CD ), Crete, paper no 561, September 2006.

[13] H. Douglas, P. Pillay, and A. K. Ziarani, "A new algorithm for transient motor current signature analysis using wavelets," IEEE Trans. Ind. Appl.,vol. 40, no. 5, pp. 1361-1368, Sep./Oct. 2004.

[14] J.A. Antonino-Daviu, M.Riera-Guasp, J.Roger Folch, M.P. Molina Palomares "Validation of a new method for the diagnosis of rotor bar failures via wavelet transform in industrial induction machines" IEEE Trans. on Industry Applications, vol. 42, no. 4pp .990-996, July/August 2006

[15] S. Elder, J.F. Watson and W.T. Thomson, "Fault detection in induction motors as a result of transient analysis" Proc. IEE 4th International Conference on Electrical Machines and Drives. London., pp. 182-186, September 1989.

[16] Toliyat, H.A., Lipo, T.A. "Transient analysis of cage induction machines under stator, rotor bar and end ring faults" IEEE Trans. on Energy Conversion,_vol 10, no 2, pp. 241 -247, June 1995.

[17] J. Penman and A. Stavrou, "Broken rotor bars: their effect on the transient performance of induction machines" IEE Proc. Electr. Power Applications. vol. 143, nº. 6, p.p 449-457, November 1996.
[18] Q.Qiu Arui, "Diagnosis of rotor fault in squirrel cage induction motors using time-varying frequency spectrum of starting stator current," in Proc. Chinese Soc. Elect. Eng., vol. 15, pp. 267-273, July 1995.

[19] R. Burnett, J.F. Watson, S. Elder, "The application of modern signal processing techniques to rotor fault detection and location within three phase induction motors," European Signal Processing Journal, Vol. 49, pp. 426-431, 1996

[20] J.F. Watson, N.C. Paterson, "Improved techniques for rotor fault detection in three-phase induction motors", The 1998 IEEE Industry Applications Conference, 1998. Thirty-Third IAS Annual Meeting, vol.1, pp. 271-277, 12-15, Oct 1998.

[21] H. Douglas, P. Pillay, and A. Ziarani, "Broken rotor bar detection in induction machines with transient operating speeds," IEEE Trans. on Energy Conversion, vol. 20, no. 1, pp. 135-141, March 2005.

[22] Z. Zhang, Z. Ren and W. Huang, "A novel detection method of motor broken rotor bars based on wavelet ridge" IEEE Transactions on Energy Conversion, Vol. 18, No. 3, pp. 417-423, September 2003.

[23] J.A.Antonino, "Diagnosis of bars breakage and other electromechanical failures in induction machines by means of the study of the startup transient using the wavelet transform," Ph.D. dissertation, Dept. Elect. Eng., Polytechnic Univ. Valencia, Valencia, Spain, Sept. 2006.

[24] W.Deleroi "Squirrel cage motor with broken bar in the rotor physical phenomena and their experimental assessment", Proc. ICEM'82. Budapest, Hungary, 1982, pp. 767-770

[25] S.Williamson, A.C.Smith "Steady state analysis of 3-phase cage motors with rotor-bar and end-ring faults" IEE Proc ., vol 129,.B, no 3 , pp. 93100, May 1982

[26] R.Polikar,'The wavelet tutorial' $\mathrm{h}$ ttp://engineering.rowan.edu/ polikar/WAVELETS/WTtutorial.html

[27] C.S. Burrus, R.A. Gopinath and H. Guo, "Introduction to Wavelets and Wavelet Transforms - a Primer", ed. Prentice Hall, 1998

[28] J.Antonino-Daviu, M.Riera-Guasp, J.Roger-Folch, F.Martínez-Jiménez, A.Peris,"Application and optimization of the discrete wavelet transform for the detection of broken rotor bars in induction machines" "Applied and Computational Harmonic Analysis" vol 21 no2 pp.268-279

[29] J.Stepina "Fundamental equations of the space vector analysis of electrical machines". Acta technica $\check{C} S A V, \mathrm{~N}^{\circ} 2,1968$, pp.184-198

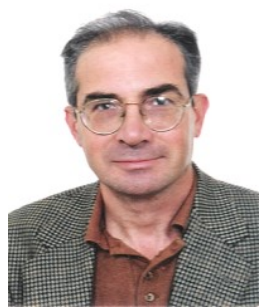

Martin Riera-Guasp received the M.Sc. degree in Industrial Engineering and the Ph.D. degree in Electrical Engineering from the Universidad Politecnica de Valencia (Spain) in 1981 and 1987, respectively. Currently he is an Associate Professor in the Department of Electrical Engineering of the Polytechnic University of Valencia. His research interests include condition monitoring of electrical machines, applications of the Wavelet Theory to electrical engineering and efficiency in electric power applications.

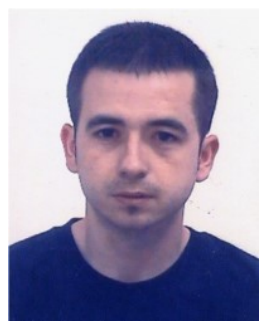

Jose A. Antonino-Daviu received the M.Sc. degree in Electrical Engineering from the Universidad Politecnica de Valencia in 2000 and the Ph.D. degree in Electrical Engineering in 2006. He worked in the private sector, being involved in several international projects. Currently, he is Associate Professor in the School of Industrial Engineering of the mentioned University, where he develops his docent and research work. His primary research interests are condition monitoring of electric machines, wavelet theory and its application to fault diagnosis and design and optimization of electrical installations and systems. 


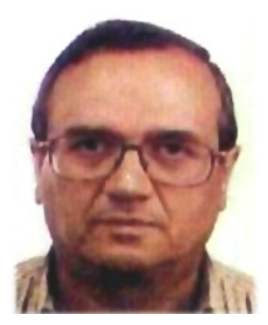

José Roger Folch obtained his M.Sc. degree in Electrical Engineering in 1970 from the Universidad Politecnica de Cataluña and his Ph.D in 1980 from the Universidad Politecnica de Valencia, Spain. From 1971 to 1978 he worked in the Electrical Industry as Project Engineer. Since 1978, he joined the Polytechnic University of Valencia and he is currently Professor of Electrical Installations and Machines. His main research areas are the Numerical Methods (F.E.M. and others) applied to the Design and Maintenance of Electrical Machines and Equipments.

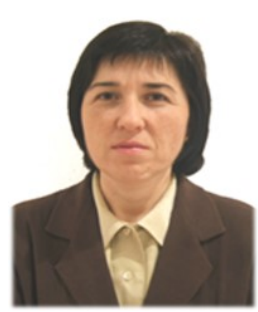

$M^{\text {a }}$ Pilar Molina Palomares received the M.S. degree in Electrical Engineering from the School of Industrial Engineering of the Universidad Politecnica de Valencia in 1990. She was Assistant Professor from 1993 to 1998. Since 1998, she is Associate Professor in the Department of Electrical Engineering of that University. Her research interests are wavelets and wavelet transform applications to fault diagnosis in transformers and electric motors. 